OPEN ACCESS

Edited by:

Michael S. Lee,

University of Minnesota Twin Cities,

United States

Reviewed by:

Essam Mohamed Elmatbouly Saber,

Benha University, Egypt

Michael C. Brodsky,

Mayo Clinic, United States

*Correspondence:

Jin-cao Chen

chenjincao2012@hotmail.com

Ke Yao

xtren@zju.edu.cn

tThese authors have contributed equally to this work

Specialty section:

This article was submitted to

Neuro-Ophthalmology,

a section of the journal

Frontiers in Neurology

Received: 21 January 2020

Accepted: 07 April 2020

Published: 15 May 2020

Citation:

Wang $Y$, Zhou $K$, Ye $Y$, Song $F$, Yu J,

Chen J and Yao K (2020) Moyamoya

Disease Associated With Morning

Glory Disc Anomaly and Other

Ophthalmic Findings: A Mini-Review.

Front. Neurol. 11:338.

doi: 10.3389/fneur.2020.00338

\section{Moyamoya Disease Associated With Morning Glory Disc Anomaly and Other Ophthalmic Findings: A Mini-Review}

\author{
Yue-ye Wang ${ }^{1 t}$, Ke-yao Zhou ${ }^{2+}$, Yang Ye ${ }^{1}$, Fan Song ${ }^{1}$, Jin Yu ${ }^{2}$, Jin-cao Chen ${ }^{2 \star}$ and \\ $\mathrm{Ke} \mathrm{Yao}{ }^{\text {* }}$ \\ ${ }^{1}$ Eye Center, Second Affiliated Hospital of Zhejiang University School of Medicine, Hangzhou, China, ${ }^{2}$ Department of \\ Neurosurgery, Zhongnan Hospital of Wuhan University, Wuhan, China
}

Moyamoya disease (MMD) is a chronic cerebrovascular disease that frequently results in intracranial ischemia or hemorrhage. Its concurrence with varying ophthalmic findings is relatively rare yet may lead to irreversible blindness. We performed a search and review of the literature to characterize the relevance of MMD (excluding moyamoya syndrome) and ophthalmic findings. As a result, a total of 38 articles identified from PubMed and Web of Science were included in this mini-review. Patients with MMD sometimes present with decreased visual acuity or visual field defects before the onset of symptomatic cerebrovascular dysfunction. The most predominant ophthalmic condition in MMD patients is the morning glory disc anomaly (MGDA). Deficiency during neuroectodermal genesis and subsequent mesodermal changes may be responsible for the association between these two diseases. Thus, it may be beneficial for patients with MGDA to receive cerebral vascular examinations as the precaution against life-threatening intracranial angiopathy. Other ophthalmic findings reported in cases of MMD include retinal vascular occlusion, optic disc pallor, cortical blindness, etc. For most of the patients with MMD, retinal examinations would be recommended to prevent potential loss of vision. It is essential for both neurologists and ophthalmologists to be aware of the correlation between cerebrovascular diseases such as MMD and ocular manifestations to achieve a comprehensive diagnosis.

Keywords: moyamoya disease, morning glory disc anomaly, neuro-ophthalmology, ocular findings, review

\section{INTRODUCTION}

Moyamoya disease (MMD) is a life-threatening cerebrovascular disease that sometimes may also lead to irreversible vision loss. The disease is characterized as progressive steno-occlusive changes occurring at the terminal portion of the internal carotid artery (ICA), initial middle cerebral artery (MCA), and anterior cerebral artery (ACA) (1). Its typical appearance on angiography of an abnormal collateral arterial network at the base of the skull resembles a puff of smoke on angiography, which is described as "moyamoya" in Japanese. MMD is an idiopathic disease, and otherwise, when patients are with moyamoya angiopathy, which is in association with other underlying diseases, e.g., neurofibromatosis or hyperthyroidism, they would be categorized as having moyamoya syndrome (2). 
Previous epidemiological studies show that the areas with high morbidity incidence of MMD are mainly East Asian countries, such as Japan, China, and Korea. According to one study in Taiwan, the prevalence of MMD in 2011 was 1.61 (per 100,000) (3). There are two peaks of the incidence, respectively, at the age of 10-20 and 35-50 (4). Ischemia and hemorrhage are predominant consequences of MMD, which mostly occur in the territories of the ICA and MCA, and sometimes present as visual symptoms onset (2). MMD relies on cerebral angiographic examinations for diagnosis and surgical revascularization for treatment, the most widely applied extracranial-intracranial bypass, for instance.

Independent ophthalmic presentations are uncommon in MMD. However, several cases of morning glory disc anomaly (MGDA) have been reported in pediatric MMD patients. The typical intracranial vascular and retinovascular anomalies suggest that there may be a common denominator between these two diseases (5). Central retinal artery occlusion, amaurosis fugax, and ocular ischemic syndrome were also reported in patients with MMD (6).

Patients who complain of visual dysfunction may be unlikely to get initially diagnosed as having potential cerebral vascular disease. Since ocular findings are usually neglected in MMD, none of those cases have been reviewed systematically. Herein, we conduct a mini-review on MMD-associated ophthalmic manifestations to provide an overall insight into its possible clinical relevance and investigate hypotheses regarding its mechanism. Since moyamoya syndrome is defined as being accompanied by correlative conditions and it would be controversial to distinguish the direct effect of this intracranial angiopathy on ophthalmic changes, we have not included any of the literature on moyamoya syndrome in this study.

\section{LITERATURE SEARCH}

We performed a search of the literature in the PubMed and Web of Science databases to identify articles related to MMD and ophthalmic findings on September 15th, 2019. The titles and abstracts of those articles were reviewed by two reviewers to confirm their quality and eligibility for further examination. The inclusion criteria were as follows: (1) MMD and ocular manifestations were simultaneously mentioned in the title or abstract, and (2) original studies or case reports. The exclusion criteria were as follows: (1) without a definite diagnosis of MMD; (2) non-English article; (3) Moyamoya syndrome; and (4) with a non-ophthalmic disease, which could probably affect visual function and not conforming to the diagnostic criteria of moyamoya syndrome. MMD was diagnosed when there was no specific underlying disease, including genetic, hereditary disorders, hematological disorders, connective-tissue diseases, infectious or chronic inflammatory conditions, metabolic diseases, and vascular injury in this study.

Abbreviations: MMD, moyamoya disease; ICA, internal carotid artery; MCA, middle cerebral artery; ACA, anterior cerebral artery; MGDA, morning glory disc anomaly; CRAO, central retinal artery occlusion; TIA, transient ischemic attack; PCA, posterior cerebral artery.
Based on the inclusion and exclusion criteria, a total of 38 eligible articles were identified and reviewed, and the year of publication ranged from 1981 to 2019.

All articles were classified according to the ophthalmic diagnosis. The various ophthalmic findings associated with MMD in these articles included MGDA, retinal vascular occlusion, nystagmus, amaurosis fugax, hypertropia, diplopia, decreased vision, visual field defect, iris hypoplasia, retinal artery tortuosity, ocular ischemia, retinochoroidal atrophy or coloboma, anterior ischemic optic neuropathy, optic nerve coloboma, optic nerve pallor, optic glioma, congenital cataract, and congenital glaucoma.

\section{AND VARIOUS OPHTHALMIC FINDINGS}

\section{Morning Glory Disc Anomaly}

According to our review, MGDA is the most frequent ophthalmic manifestation in all MMD patients included. MGDA is a rare congenital deficiency of the optic disc characterized by an enlarged, funnel-shaped excavation of the posterior pole involving the hypogenetic optic disc, which resembles a morning glory flower. Generally, this malformation is considered to be unilateral, while bilateral involvements were occasionally reported. Patients of MGDA mainly present with decreased visual acuity at the onset due to dysplasia of the optic disc. This disease is associated with various conditions, including intracranial vascular abnormalities; mid-line facial abnormalities; 47, XYY syndrome; Down syndrome; etc. (7-9).

We identified 13 articles of MMD-associated MGDA, including 11 case reports, 1 ophthalmic image report, and 1 retrospective study $(5,9-20)$. The retrospective study evaluated intracranial vascular anomalies in 20 patients with MGDA and diagnosed four of them with MMD (9). The ophthalmic images published in JAMA Ophthalmology in 2015 showed fundus and ultrasonography of an MGDA patient. The compensatory collateralization of chorioretinal anastomoses in this patient also imparted a "moyamoya" bypass system, indicating that MGDA may have pathological correlations with MMD (11).

The characteristics of the 11 cases are listed in (Table 1). Of all the patients included, 6 (54.5\%) were male; most of them were children, with a mean age of 9.7 years (median 5 , range $2-29$ ). A total of $9(81.8 \%)$ patients were diagnosed with unilateral MGDA, while only $2(18.2 \%)$ were diagnosed with bilateral MGDA. Among all the $8(72.7 \%)$ bilateral MMD patients, 7 had unilateral MGDA (left eye: 5, right eye: 2), and only one had bilateral MGDA. The remaining two patients with unilateral MMD were associated with ipsilateral MGDA, while the other patient with unilateral MMD was associated with bilateral MGDA. Generally, MDGA is unilaterally presented, while MMD is bilaterally presented, yet the severity can differ between sides. Therefore, it would be logical that the most frequent situation in patients is with bilateral MMD and unilateral MGDA. Interestingly, patients with unilateral MMD only presented MGDA on the same side in current cases, the situation of these two diseases with opposite laterality has not been described yet. Besides, 
TABLE 1 | Cases of Moyamoya disease associated with morning glory disc anomaly.

\begin{tabular}{|c|c|c|c|c|c|c|}
\hline No. & Author & Year & Age & Gender & Diagnosis & Intracranial vessels \\
\hline Case 1 & Sathyan, S. & 2018 & 16 & Female & $\begin{array}{l}\text { MGDA (OS); high myopia (OS); } \\
\text { bilateral MMD }\end{array}$ & $\begin{array}{l}\text { Left ICA stenosis; (right side } \\
\text { post-operation) }\end{array}$ \\
\hline Case 3 & Loddenkemper, T. & 2008 & 2 & Female & $\begin{array}{l}\text { MGDA (OU); bilateral MMD; } \\
\text { pituitary stalk duplication }\end{array}$ & $\begin{array}{l}\text { Bilateral ICA and MCA stenosis; } \\
\text { right ACA stenosis }\end{array}$ \\
\hline Case 5 & Sabti, K. & 2005 & 3 & Male & $\begin{array}{l}\text { MGDA (OU); left MMD; Congenital } \\
\text { third nerve palsy }\end{array}$ & Left ICA stenosis \\
\hline Case 6 & Quah, B. L. & 2005 & 4 & Male & $\begin{array}{l}\text { MGDA (OS); bilateral MMD; } \\
\text { midline cranial defects }\end{array}$ & $\begin{array}{l}\text { Right ICA and MCA stenosis; left } \\
\text { ICA occlusion }\end{array}$ \\
\hline Case 7 & Taskintuna, I. & 2003 & 4 & Male & $\begin{array}{l}\text { MGDA (OD); bilateral MMD; } \\
\text { persistent hyaloid artery remnant; } \\
\text { midline cranial defects }\end{array}$ & $\begin{array}{l}\text { Bilateral ICA stenosis; bilateral } \\
\text { MCA M1 segment stenosis and } \\
\text { occlusion }\end{array}$ \\
\hline Case 10 & Bakri, S. J. & 1999 & 10 & Female & $\begin{array}{l}\text { MGDA (OD); bilateral MMD; } \\
\text { chorioretinal coloboma (OS); } \\
\text { sphenopharyngeal } \\
\text { meningoencephalocele }\end{array}$ & Bilateral MCA stenosis \\
\hline Case 11 & Massaro, M. & 1998 & 5 & Male & $\begin{array}{l}\text { MGDA (OS); left MMD; retinal } \\
\text { detachment (OS) }\end{array}$ & $\begin{array}{l}\text { Left ICA, and its bifurcation into } \\
\text { MCA and ACA stenosis }\end{array}$ \\
\hline
\end{tabular}

MMD, Moyamoya disease; MGDA, morning glory disc anomaly; OS, oculus sinister; OD, oculus dexter; OU, oculus uterque; ICA, internal carotid artery; MCA, middle cerebral artery; $A C A$, anterior cerebral artery.

$3(27.3 \%)$ patients were found with midline cranial defects, 2 (18.2\%) patients with choroidal coloboma, and 2 (18.2\%) patients with meningoencephalocele. The involvement of ICA in cerebral angiography was most prevalent, found in 10 (90.9\%) patients, while MCA involvement was found in $6(54.5 \%)$ patients and ACA involvement was found in $3(27.3 \%)$ patients.

Most of the patients in (Table 1) were also diagnosed with other congenital abnormalities such as midline cranial defect or duplication of the pituitary stalk, reflecting mutual fetal dysgenesis to be the possible underlying cause. Komiyama, $\mathrm{M}$ once postulated that MMD is a vascular form of neurocristopathy. The finding that the steno-occlusive changes of MMD only exist in the cerebral arteries originating from the neural crest fits in this context (21). According to the mainstream hypothesis, MGDA is the consequence of primary neuroectodermal dysgenesis, characteristically with central gliosis and vascular damage at the optic disc (22). The pathological changes involving the optic disc and intracranial vessels are suggestive of a similar deficiency during ectoderm development in both diseases.

The risk of intracranial vascular anomalies was higher in MGDA patients than in non-MGDA patients, according to Lenhart's study (9). This study reviewed and evaluated neurologic anomalies in 20 MGDA patients and 40 non-MGDA patients as a control. As a result, cerebral anomalies were more common in patients with MGDA, 9 of 20 (45\%) compared with 10 in 40
(25\%) in the control group. The intracranial vascular anomalies in these patients ranged within a spectrum, from only mild congenital variants to progressive stenosis or occlusion, as the most severe condition to be MMD. MMD was diagnosed in 3 of 20 patients with MGDA, while it was diagnosed in none of the control. The estimated prevalence of MMD in MGDA patients was $15 \%$ in this study, significantly higher than that in the whole population. Nevertheless, the prevalence in this study is deduced within a limited sample size, and further study on a larger scale is still required.

Interestingly, a cross-sectional study analyzed the retinal morphology of MMD patients by optical coherence tomography. In patients without symptomatic retinal abnormalities, a significant reduction in optic nerve head volume and a thinner macular retinal nerve fiber layer were revealed, similar to the characteristics of MGDA. The authors indicated that both MMD and MGDA might involve neuroectodermal dysgenesis and subsequent mesodermal changes (23), even if it was not able to be distinguished in fundus photography or diagnosed as retinopathy, the volume of optic nerve head was with detectable change. Abnormal retinal findings in MGDA may be indications of intracranial vascular pathogenesis as the consequence of mutual mesenchymal dysgenesis, and optical coherence tomography is a simple and non-invasive technique to achieve early diagnosis precede the clinical manifestation of MMD. Patients with decreased optic nerve head volume or thinning 
TABLE 2 | Cases of Moyamoya disease associated with retinal vascular occlusion and other ophthalmic findings.

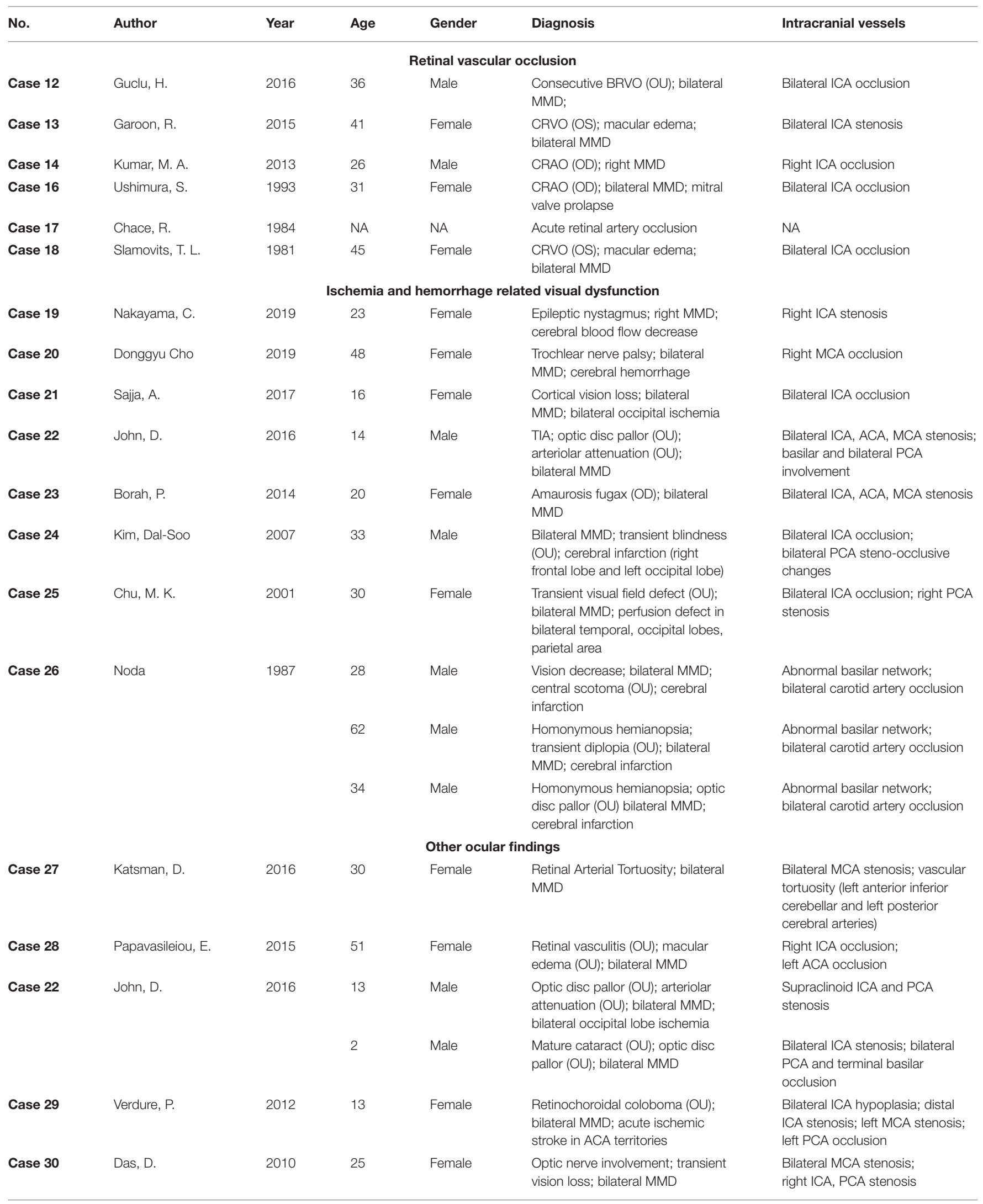


TABLE 2 | Continued

\begin{tabular}{|c|c|c|c|c|c|c|}
\hline No. & Author & Year & Age & Gender & Diagnosis & Intracranial vessels \\
\hline Case 31 & Johnson, S. M. & 2009 & 16 & Female & $\begin{array}{l}\text { Congenital glaucoma; bilateral } \\
\text { MMD; myopia }\end{array}$ & $\begin{array}{l}\text { Bilateral MCA; left PCA } \\
\text { involvements }\end{array}$ \\
\hline Case 32 & Spengos, K. & 2008 & 42 & Female & $\begin{array}{l}\text { Optic Nerve Coloboma (OS); left } \\
\text { MMD }\end{array}$ & $\begin{array}{l}\text { Anterior and posterior cerebral } \\
\text { circulation }\end{array}$ \\
\hline Case 33 & Chen, C. S. & 2007 & 51 & Female & $\begin{array}{l}\text { Non-arteritic anterior ischemic } \\
\text { optic neuropathy; bilateral MMD }\end{array}$ & $\begin{array}{l}\text { Right ICA occlusion; } \\
\text { left ICA stenosis }\end{array}$ \\
\hline \multirow[t]{2}{*}{ Case 34} & Khan, N. & 2004 & 4 & Female & $\begin{array}{l}\text { Iris hypoplasia and fixed dilated } \\
\text { pupils (OU); bilateral MMD; } \\
\text { bilateral dolichoectatic internal } \\
\text { carotid arteries; patent ductus } \\
\text { arteriosus }\end{array}$ & $\begin{array}{l}\text { Bilateral ICA stenosis; left ACA } \\
\text { occlusion }\end{array}$ \\
\hline & & & 6 & Male & $\begin{array}{l}\text { Iris hypoplasia and fixed dilated } \\
\text { pupils }(\mathrm{OU}) \text {; bilateral MMD; patent } \\
\text { ductus arteriosus }\end{array}$ & $\begin{array}{l}\text { Bilateral ICA stenosis; left MCA } \\
\text { stenosis; left ACA aplasia }\end{array}$ \\
\hline Case 35 & Harissi-Dagher, M. & 2004 & 20 & Female & $\begin{array}{l}\text { Chorioretinal atrophy }(\mathrm{OU}) \text {; } \\
\text { bilateral MMD }\end{array}$ & Bilateral ICA occlusion \\
\hline Case 26 & Noda & 1987 & 6 & Male & $\begin{array}{l}\text { Amaurosis fugax (OU); concentric } \\
\text { construction of visual field }(\mathrm{OU}) \\
\text { bilateral MMD }\end{array}$ & $\begin{array}{l}\text { Abnormal basilar network; } \\
\text { bilateral carotid artery occlusion }\end{array}$ \\
\hline \multirow[t]{2}{*}{ Case 36} & Okuno, T. & 1985 & 5 & Female & Optic glioma (OU); bilateral MMD & Bilateral carotid systems \\
\hline & & & 7 & Female & Optic glioma; bilateral MMD & Bilateral ICA \\
\hline
\end{tabular}

MMD, Moyamoya disease; MGDA, morning glory disc anomaly; BRVO, branch retinal vein occlusion; CRVO, central retinal vein occlusion; CRAO, central retinal artery occlusion; OS, oculus sinister; $O D$, oculus dexter; OU, oculus uterque; ICA, internal carotid artery; MCA, middle cerebral artery; ACA, anterior cerebral artery; PCA, posterior cerebral artery; NA, not available.

macular retinal nerve fiber layer should receive neuroradiological examinations for the diagnosis of any potential cerebral vascular disease. Those patients have not been conclusively shown to have the same risk for intracranial vascular occlusion, though the association should not be underestimated. Most of them may only present with mild vascular anomalies. Thus, the indication of preemptive treatment with intracranial vascular bypass is limited in this subgroup, and further discussion with a neurosurgeon would be recommended.

\section{Retinal Vascular Occlusion}

Obstruction of retinal vessels, particularly the central retinal artery, has been reported in several cases of MMD patients. A total of 6 cases reporting MMD associated retinal vascular occlusion are reviewed in this study (Table 2) (24-29). The chief complaint of almost all patients was acute vision loss. One patient also complained of a severe headache, while two patients were found with a relative afferent pupillary defect. The age of those patients ranged from 25 to 40 years old, in accordance with the second age peak of onset of MMD.

Retinal vascular occlusion can be recurrent in MMD patients. The patient in case 12 was diagnosed with bilateral MMD and received encephalo-myo-synangiosis as a surgical intervention. Six years after the surgery, the patient complained of decreased vision in the right eye, and branch retinal vein occlusion was found in this eye. Recurrence occurred in his contralateral eye 12 years later (24). Case 13 indicated that the treatment of MMD might provide an increased blood supply to the ophthalmic artery and improve retinal perfusion as a possible solution to rescue vision (25). The visual acuity of this patient was significantly improved from $20 / 200$ to $20 / 25$ after an uncomplicated superficial temporal artery-MCA anastomosis. Case 16 reported a pregnant patient of mitral valve prolapse combined with right eye central retinal artery occlusion (CRAO) and bilateral MMD (27). It is considered challenging to identify a mechanism of CRAO in MMD patients, which can be caused by complicated factors and interactions. The hypercoagulable state during pregnancy, the pathological consequence of MMD, and the emboli from mitral valve prolapse may all be presumably responsible for this case.

The steno-occlusive changes of MMD usually occur at the supraclinoidal portion of the ICA and its two main branches. In some circumstances, the slow progression of MMD allows collateral circulation from the external carotid artery to develop and supply the central retinal artery (30). Aside from patients with cardiovascular emboli, CRAO is generally seen in the elderly population, especially those over 50 years old. However, the average age in the cases of MMD-associated CRAO mentioned above showed a younger tendency (26). When steno-occlusive and chronic ischemic changes involve the ophthalmic artery in MMD patients without the formation of collateral circulation, though rare, retinal artery occlusion may eventually be a complication. As for retinal vein occlusion in MMD, whether it results from the intravascular inflammatory change and subsequent compression of the vein, or it is coincidental is still in controversy (24).

\section{Ischemia- and Hemorrhage-Related Visual Disturbance}

Diverse ocular manifestations are sometimes consequences of ischemia or hemorrhage in MMD and may differ from the regions involved. A total of 10 cases from eight articles referring 
to ischemia- or hemorrhage-related ocular manifestations are summarized and reviewed, five of which are from case series (Table 2) (31-38). Most patients had a significant ischemia lesion in the cerebral imaging, and only one patient was diagnosed as having a hemorrhage. One patient in Case 23 did not have any suggestive change of ischemia or hemorrhage in imaging. She was found with recurrent amaurosis fugax, and cerebral angiography only showed bilateral narrowing of the ICA, ACA, and MCA (35).

Decreased cerebral blood flow is a prevalent pathological change in MMD with a tendency to cause cerebral hypoperfusion. Severe hypoperfusion may lead to cognitive impairment and intellectual disability (4). Case 19 reported a patient with cerebral hypoperfusion-related epilepsy and nystagmus. She complained of headache and transient hemiparesis on the left side and was subsequently diagnosed with right-side MMD, grade III of Suzuki's angiographic staging. Hypoperfusion in her right basal ganglia, frontal, and parietal regions were also found. After a hyperventilation test, which is a trigger of transient ischemic attack (TIA) in MMD patients, she deteriorated with new-onset epileptic nystagmus. In this case, the authors indicated that the decreased cerebral blood flow in the right frontal region was responsible for the epileptic nystagmus.

Hemorrhage may be a result of aberrant angiogenesis, such as fragile microaneurysms or false aneurysms, especially those occurring in collateral vessels of MMD (39). Case 20 described a patient who used to have an intracranial hemorrhage. Even after receiving a superficial temporal artery to MCA bypass, he still suffered from diplopia and hypertropia after the operation. His hypertropia and diplopia were considered as consequences of trochlear nerve palsy, for the past hemorrhage had damaged the right trochlear nucleus and intra-axial trochlear nerve (32).

Although posterior circulation is rarely involved in MMD, it should still be regarded as an alternative cause of acute vision decrease. The possible mechanism of posterior circulation involvement in MMD is similar to that of anterior circulation besides an isolated pathological change, the unstable hemodynamic state of the posterior cerebral artery (PCA) (37). Patients with PCA involvement are usually related to more severe conditions and worse prognosis $(4,40)$. Prior studies have reported that $\sim 29 \%$ of $\mathrm{MMD}$ patients were with PCA involvement, and $17 \%$ of them had an infarction in territories of PCA (4). Patients with PCA involvement usually present with specific symptoms related to impaired vision. Steno-occlusion changes in the posterior choroidal arteries of deep PCA can result in sectoranopia, while superficial segments infarction of PCA frequently leads to visual field defects (41). According to our review, 12 cases of PCA involvement were identified with ocular abnormalities, including optic disc pallor, congenital cataract, transient visual field defect, and occipital lobe infarction-related cortical blindness $(33,34,36,37)$. Most of these patients received revascularization surgery, such as superficial temporal artery to MCA bypass, encephalo-duro-galeo-synangiosis, etc. However, the therapeutic effect on visual manifestations was uncertain. Three patients in case 26 experienced vision improvement, yet cortical blindness persisted in the patient of case 21 . The regions of ischemic stroke, time from occurrence to surgery, territories of the cerebral vessels with the steno-occlusive change, and the general condition of patients may all be implicit factors in determining the therapeutic effect.

\section{Other Ocular Findings}

Other uncommon ocular findings in MMD patients are referred to in this section from 12 articles, including optic nerve involvement, congenital cataract, and retinochoroidal anomaly (Table 2) (34, 38, 42-51). In Case 31, a 13-year-old female patient was diagnosed with congenital glaucoma and MMD. The author pointed out an overlapped genetic mutation responsible for moyamoya angiopathy, as well as Fanconi anemia, congenital glaucoma, and other congenital abnormalities (46). Case 34 reported two pediatric MMD patients with bilateral iris hypoplasia and patent ductus arteriosus (49). Various congenital diseases are suggested that simultaneously involve cerebral vascular dysplasia, cardiovascular malformations, and ocular anomalies. Further study is needed to focus on possible target genes responsible for the conditions that related to MMD, congenital ophthalmic diseases, and other congenital diseases.

Retinal vasculature is a microcirculation that can be noninvasively observed by fundus photography. It could also be considered as a reflection of the systemic circulation. Case 27 reported a 30-year-old female with headache and blurred vision who was diagnosed with retinal arterial tortuosity and bilateral MMD. Proangiogenic factor levels increase as compensation and lead to neovascularization, which is hypothesized as an underlying explanation for the formation of retinal vascular tortuosity in this case (42). Case 28 described a patient of ocular ischemic syndrome combined with MMD and received a graft surgery. Significant damage to the blood-retinal barrier persists despite postoperative cerebral reperfusion. Though the ocular ischemia is capable of being relieved after the surgery of MMD with improved retinal circulation times, visual acuity outcomes are in limited optimism (43). Some patients with MMD are still at the risk of having retinovascular abnormalities, yet without obvious ocular symptoms. J.I. Chung reported a case of an MMD patient with ophthalmic rete mirabile, a plexiform vascular network of a reconstituted ophthalmic artery which is rarely seen in humans (52). The complex of the abnormal ophthalmic artery and its collaterals could be considered as embryonic remnant anastomosis and annexations and, thus, to some extent, explain the cause of prenatal cerebral vascular occlusion in MMD.

\section{CONCLUSION}

MMD is associated with varying ophthalmic findings, particularly MGDA, according to previous studies. The risk of having MMD in patients of MGDA is higher than in the general population. Deficiency during neuroectodermal genesis and the subsequent mesodermal changes may be responsible for this association. Cerebral vascular examinations are recommended for MGDA patients to exclude any potential life-threatening intracranial vascular diseases. Other ophthalmic findings related to MMD include retinal vascular occlusion, visual field defects caused by ischemia or hemorrhage, optic disc abnormalities, etc. Retinal examinations would be beneficial for MMD patients to prevent severe loss of vision. Posterior circulation involvement in 
MMD is rare but should not be underestimated, for sometimes it is responsible for acute or transient vision loss. Both neurologists and ophthalmologists should recognize the correlation between ocular manifestations and cerebrovascular diseases such as $\mathrm{MMD}$, in order to achieve a comprehensive diagnosis.

\section{AUTHOR CONTRIBUTIONS}

KY, JC, and YW designed this review. YW and KZ drafted the manuscript and searched and reviewed the database and all included articles. YY and FS reviewed the articles

\section{REFERENCES}

1. Zhang H, Zheng L, Feng L. Epidemiology, diagnosis and treatment of moyamoya disease. Exp Ther Med. (2019) 17:1977-84. doi: $10.3892 /$ etm.2019.7198

2. Scott RM, Smith ER. Moyamoya disease and moyamoya syndrome. $N$ Engl J Med. (2009) 360:1226-37. doi: 10.1056/NEJMra0804622

3. Chen PC, Yang SH, Chien KL, Tsai IJ, Kuo MF. Epidemiology of moyamoya disease in Taiwan: a nationwide population-based study. Stroke. (2014) 45:1258-63. doi: 10.1161/STROKEAHA.113.004160

4. Fujimura M, Bang OY, Kim JS. Moyamoya Disease. Front Neurol Neurosci. (2016) 40:204-20. doi: 10.1159/000448314

5. Sathyan S, Chackochan M. Morning glory disc anomaly and facial hemangiomas in a girl with moyamoya syndrome. Indian J Ophthalmol. (2018) 66:1644-6. doi: 10.4103/ijo.IJO_538_18

6. "Ocular Manifestations of Moyamoya Disease", Eye Wiki, American Academy of Ophthalmology. (2019). Available online at: https://eyewiki.org/w/index. php?title=Ocular_manifestations_of_Moyamoya_Disease\&oldid $=51655$ (accessed January 13, 2020).

7. Georgalas I, Spyropoulos D, Paraskevopoulos T, Rotsos T. Morning Glory Disc Anomaly in a Child with Esotropia. J Pediatr. (2018) 203:458.el. doi: 10.1016/j.jpeds.2018.06.014

8. Thoma D, Nijs I, Demaerel P, Casteels I. Morning glory disc anomaly with an ipsilateral enlargement of the optic nerve pathway. Eur J Paediatr Neurol. (2017) 21:787-91. doi: 10.1016/j.ejpn.2017.04.1334

9. Lenhart PD, Lambert SR, Newman NJ, Biousse V, Atkinson DS Jr, Traboulsi EI, et al. Intracranial vascular anomalies in patients with morning glory disk anomaly. Am J Ophthalmol. (2006) 142:644-50. doi: 10.1016/j.ajo.2006.05.040

10. Ponnatapura J. Morning glory syndrome with Moyamoya disease: a rare association with role of imaging. Indian J Radiol Imaging. (2018) 28:165-8. doi: 10.4103/ijri.IJRI_219_17

11. Brodsky MC, Parsa CF. The moyamoya optic disc. JAMA Ophthalmol. (2015) 133:164. doi: 10.1001/jamaophthalmol.2014.602

12. Loddenkemper T, Friedman NR, Ruggieri PM, Marcotty A, Sears J, Traboulsi EI. Pituitary stalk duplication in association with moya moya disease and bilateral morning glory disc anomaly - broadening the clinical spectrum of midline defects. J Neurol. (2008) 255:885-90. doi: 10.1007/s00415-008-0799-5

13. Williams M, Adas A, Sharma N, Gibson M. Moyamoya disease presenting to the ophthalmology clinic. Can J Ophthalmol. (2006) 41:633-4. doi: 10.1016/S0008-4182(06)80038-4

14. Sabti K, Hajj BA, Hwang JM, Traboulsi EI, Reid J. Congenital third nerve palsy, moyamoya disease and optic nerve head staphyloma. Br J Ophthalmol. (2005) 89:778-9. doi: 10.1136/bjo.2004.059246

15. Quah BL, Hamilton J, Blaser S, Heon E, Tehrani NN. Morning glory disc anomaly, midline cranial defects and abnormal carotid circulation: an association worth looking for. Pediatr Radiol. (2005) 35:525-8. doi: 10.1007/s00247-004-1345-y

16. Taskintuna I, Oz O, Teke MY, Kocak H, Firat E. Morning glory syndrome: association with moyamoya disease, midline cranial defects, central nervous system anomalies, and persistent hyaloid artery remnant. Retina. (2003) 23:400-2. doi: 10.1097/00006982-200306000-00018 based on inclusion and exclusion criteria. YW, KZ, YY, FS, JY, JC, and KY provided comments and revised the manuscript. All authors have approved the final version of the manuscript.

\section{ACKNOWLEDGMENTS}

We acknowledge the Eye Center, Second Affiliated Hospital of Zhejiang University School of Medicine, as well as the Department of Neurosurgery, Zhongnan Hospital of Wuhan University.

17. Krishnan C, Roy A, Traboulsi E. Morning glory disk anomaly, choroidal coloboma, and congenital constrictive malformations of the internal carotid arteries (moyamoya disease). Ophthalmic Genet. (2000) 21:21-4. doi: 10.1076/1381-6810(200003)2111-IFT021

18. Komiyama M, Yasui T, Sakamoto H, Fujita K, Sato T, Ota M, et al. Basal meningoencephalocele, anomaly of optic disc and panhypopituitarism in association with moyamoya disease. Pediatr Neurosurg. (2000) 33:100-4. doi: $10.1159 / 000028984$

19. Bakri SJ, Siker D, Masaryk T, Luciano MG, Traboulsi EI. Ocular malformations, moyamoya disease, and midline cranial defects: a distinct syndrome. Am J Ophthalmol. (1999) 127:356-7. doi: 10.1016/S0002-9394(98)00317-1

20. Massaro M, Thorarensen O, Liu GT, Maguire AM, Zimmerman RA, Brodsky MC. Morning glory disc anomaly and moyamoya vessels. Arch Ophthalmol. (1998) 116:253-4.

21. Komiyama M. Moyamoya disease is a vascular form of neurocristopathy: disease of the embryologic cephalic neural crest. Childs Nerv Syst. (2017) 33:567-8. doi: 10.1007/s00381-017-3369-2

22. Romano F, Giuffre C, Arrigo A, Corbelli E, Battaglia Parodi M, Bandello F. Case report: optical coherence tomography angiography in morning glory disc anomaly. Optom Vis Sci. (2018) 95:550-2. doi: 10.1097/OPX.0000000000001225

23. Albrecht P, Blasberg C, Lukas S, Ringelstein M, Muller AK, Harmel $\mathrm{J}$, et al. Retinal pathology in idiopathic moyamoya angiopathy detected by optical coherence tomography. Neurology. (2015) 85:521-7. doi: 10.1212/WNL.0000000000001832

24. Guclu H, Gurlu VP, Ozal SA, Esgin H. A Moyamoya patient with bilateral consecutive branch retinal vein occlusion. Neuroophthalmology. (2016) 40:936. doi: 10.3109/01658107.2016.1148174

25. Garoon R, Carvounis PE. Central retinal vein occlusion with bilateral stenosis of the internal carotid arteries. Lancet. (2015) 385:914. doi: 10.1016/S0140-6736(15)60124-6

26. Kumar MA, Ganesh BA. CRAO in Moyamoya Disease. J Clin Diagn Res. (2013) 7:545-7. doi: 10.7860/JCDR/2013/4579.2819

27. Ushimura S, Mochizuki K, Ohashi M, Ito S, Hosokawa H. Sudden blindness in the fourth month of pregnancy led to diagnosis of moyamoya disease Ophthalmologica. (1993) 207:169-73. doi: 10.1159/000310428

28. Chace R, Hedges TR III. Retinal artery occlusion due to moyamoya disease. J Clin Neuroophthalmol. (1984) 4:31-4.

29. Slamovits TL, Klingele TG, Burde RM, Gado MH. Moyamoya disease with central retinal vein occlusion. Case report. J Clin Neuroophthalmol. (1981) 1:123-7.

30. Robert T, Ciccio G, Sylvestre P, Chiappini A, Weil AG, Smajda S, et al. Anatomic and angiographic analyses of ophthalmic artery collaterals in moyamoya disease. AJNR Am J Neuroradiol. (2018) 39:1121-6. doi: 10.3174/ajnr.A5622

31. Nakayama C, Mikami T, Ukai R, Chiba R, Enatsu R, Suzuki H, et al. Moyamoya disease with epileptic nystagmus: a case report. J Clin Neurosci. (2019) 70:251-4. doi: 10.1016/j.jocn.2019.08.069

32. Cho D, Walker FO, Ryu BJ. Trochlear nerve palsy associated with Moyamoya disease. J Clin Neurosci. (2019) 66:266-8. doi: 10.1016/j.jocn.2019.05.046 
33. Sajja A, Tsering D, Mooser AC, DeFreitas TA, Carpenter J, Magge SN. Patient with severe moyamoya disease who presents with acute cortical blindness. Stroke. (2017) 48:e126-9. doi: 10.1161/STROKEAHA.116.015548

34. John D, Muthusamy K, Bandla B, Sudhakar SV, Thomas M. Ocular features and visual outcome in children with moyamoya disease and moyamoya syndrome: a case series. J Clin Diagn Res. (2016) 10:NR01-4. doi: 10.7860/JCDR/2016/19153.7744

35. Borah P, Sharma V, Basumatary LJ, Das M, Goswami M, Kayal AK. Varied presentations of moyamoya disease in a tertiary care hospital of north-east India. Ann Indian Acad Neurol. (2014) 17:317-20. doi: 10.4103/0972-2327.138518

36. Kim DS, Kang SG, Yoo DS, Huh PW, Cho KS, Kim MC. Sudden cortical blindness in an adult with moyamoya disease. Surg Neurol. (2007) 67:303-7. doi: 10.1016/j.surneu.2006.03.043

37. Chu MK, Lee IH, Kim DI, Kim SM. Moyamoya disease initially presenting visual field defect. Yonsei Med J. (2001) 42:566-70. doi: 10.3349/ymj.2001.42.5.566

38. Noda S, Hayasaka S, Setogawa T, Matsumoto S. Ocular symptoms of moyamoya disease. Am J Ophthalmol. (1987) 103:812-6. doi: 10.1016/S0002-9394(14)74399-5

39. Kim JS. Moyamoya disease: epidemiology, clinical features, and diagnosis. $J$ Stroke. (2016) 18:2-11. doi: 10.5853/jos.2015.01627

40. Shang S, Zhou D, Ya J, Li S, Yang Q, Ding Y, et al. Progress in moyamoya disease. Neurosurg Rev. (2018) 43:371-82. doi: 10.1007/s10143-018-0994-5

41. Cereda C, Carrera E. Posterior cerebral artery territory infarctions. Front Neurol Neurosci. (2012) 30:128-31. doi: 10.1159/000333610

42. Katsman D, Klufas MA, Sarraf D, Sadda S. Retinal arterial tortuosity in moyamoya disease. JAMA Ophthalmol. (2016) 134:111-4. doi: 10.1001/jamaophthalmol.2015.4645

43. Papavasileiou E, Sobrin L, Papaliodis GN. Ocular ischemic syndrome presenting as retinal vasculitis in a patient with moyamoya syndrome. Retin Cases Brief Rep. (2015) 9:170-2. doi: 10.1097/ICB.00000000000 00129

44. Verdure P, Le Moigne O, Massardier EG, Vanhulle C, Tollard E, Maltete D. Migraine-like headache and ocular malformations may herald Moyamoya syndrome. Rev Neurol. (2012) 168:460-1. doi: 10.1016/j.neurol.2011. 09.011
45. Das D, Handique SK, Bhattacharjee H, Buragohain SK, Bharali G, Deka AC, et al. Moyamoya disease with neuro-ophthalmic manifestations: a rare case report. Eye Brain. (2010) 2:63-5. doi: 10.2147/EB.S7614

46. Johnson SM, Stanley L. Moyamoya in association with congenital glaucoma. Clin Pediatr. (2009) 48:202-5. doi: 10.1177/0009922808323904

47. Spengos K, Vassilopoulou S, Anagnostou E, Paraskevas G, Zis VP. Moyamoya syndrome associated with optic nerve coloboma and mental retardation. Cerebrovasc Dis. (2008) 26:448-9. doi: 10.1159/000157631

48. Chen CS, Lee AW, Kelman S, Wityk R. Anterior ischemic optic neuropathy in moyamoya disease: a first case report. Eur J Neurol. (2007) 14:823-5. doi: 10.1111/j.1468-1331.2007.01819.x

49. Khan N, Schinzel A, Shuknecht B, Baumann F, Ostergaard JR, Yonekawa Y. Moyamoya angiopathy with dolichoectatic internal carotid arteries, patent ductus arteriosus and pupillary dysfunction: a new genetic syndrome? Eur Neurol. (2004) 51:72-7. doi: 10.1159/000076248

50. Harissi-Dagher M, Sebag M, Dagher JH, Moumdjian R. Chorioretinal atrophy in a patient with moyamoya disease. Case report. J Neurosurg. (2004) 101:8435. doi: 10.3171/jns.2004.101.5.0843

51. Okuno T, Prensky AL, Gado M. The moyamoya syndrome associated with irradiation of an optic glioma in children: report of two cases and review of the literature. Pediatr Neurol. (1985) 1:311-6. doi: 10.1016/0887-8994(85)90036-0

52. Chung JI, Weon YC. Ophthalmic rete mirabile: the first angiographic documentation of embryonic ophthalmic collaterals in a patient with moyamoya disease. A case report. Interv Neuroradiol. (2008) 14:293-6. doi: $10.1177 / 159101990801400309$

Conflict of Interest: The authors declare that the research was conducted in the absence of any commercial or financial relationships that could be construed as a potential conflict of interest.

Copyright (C) 2020 Wang, Zhou, Ye, Song, Yu, Chen and Yao. This is an open-access article distributed under the terms of the Creative Commons Attribution License (CC $B Y)$. The use, distribution or reproduction in other forums is permitted, provided the original author(s) and the copyright owner(s) are credited and that the original publication in this journal is cited, in accordance with accepted academic practice. No use, distribution or reproduction is permitted which does not comply with these terms. 